\title{
Material leñoso de gran tamaño en dos cuencas de la Cordillera de la Costa de Chile con diferente historia de uso del suelo
}

\author{
Large wood in two catchments from the Coastal Mountain range with different land use history \\ Héctor Ulloa a*, Andrés Iroumé b, Mario A Lenzi c, Andrea Andreoli d, Cristián Álvarez e, Víctor Barrera ${ }^{\text {b }}$ \\ *Autor de correspondencia: ${ }^{a}$ Universidad Austral de Chile, Facultad de Ciencias Forestales y Recursos Naturales, \\ Escuela de Graduados, casilla 567, Valdivia, Chile, ulloacontreras@gmail.com

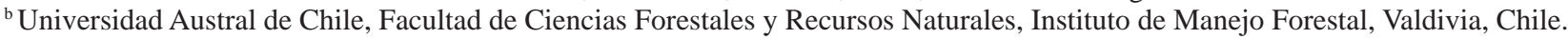 \\ ' Università degli studi di Padova, viale dell’Università, Legnaro, Italy. \\ ${ }^{\mathrm{d}}$ Universidad de Concepción, Facultad de Ciencias Forestales, Departamento Manejo de Bosques y Medio Ambiente, \\ Concepción, Chile. \\ e Universidad Austral de Chile, Facultad de Ciencias, Instituto de Física, Valdivia, Chile.
}

\section{SUMMARY}

Previous researches indicated that in-stream large wood (LW) depended on the characteristics of the riparian vegetation. This is the first study about LW abundance, longitudinal distribution and sediment trapped by large wood structures comparing two stream segments in catchments with contrasting land use, located in the Coastal Mountain Range, Southern Chile. One such catchment (Vuelta de Zorra) is covered mainly by native forests and the other (Pichún) runs under productive plantations. In a stream segment of each catchment the riparian vegetation was characterized, each LW piece was measured and geo-referenced and the channel morphology was defined. Vegetation along the Vuelta de Zorra channel corresponded to an adult second growth forest $c a$. 200 years old with a basal area of $69 \mathrm{~m}^{2} \mathrm{ha}^{-1}$; while in Pichún, it was a much degraded remnant of old native forests with a basal area of $24 \mathrm{~m}^{2} \mathrm{ha}^{-1}$. Considering channel bankfull area as reference, LW volume at Vuelta de Zorra and Pichún was 109 and $56 \mathrm{~m}^{3} \mathrm{ha}^{-1}$, respectively. Trapped sediment values in the stream segments were 82 and $30 \mathrm{~m}^{3} \mathrm{~km}^{-1}$ for Vuelta de Zorra and Pichún, respectively; in both channels the accumulations of LW played a key role in the amount of trapped sediment. LW volume was associated with the characteristics of the riparian vegetation which is a consequence of the history of forest interventions and land uses in each catchment.

Key words: abundance, longitudinal distribution, mobility, mountain channels.

\section{RESUMEN}

El material leñoso en los cauces de montaña depende, principalmente, de las características del bosque ribereño que los alimenta. Esta investigación presenta el primer reporte sobre distribución longitudinal, abundancia y la propiedad de atrapar sedimento de fondo, del material leñoso en dos cuencas con uso contrastante del suelo, localizadas en la Cordillera de la Costa del sur de Chile. La cuenca Vuelta de Zorra ( $39^{\circ} 58^{`} 12 \mathrm{~S}$; $73^{\circ} 34^{`} 13^{\prime}$ O) está cubierta principalmente por bosque nativo y la Pichún ( $37^{\circ} 30^{`} 12^{`}$ S; $66^{\circ} 45^{`}$ $52^{`} \mathrm{O}$ ) ha estado sometida a rotaciones sucesivas de plantaciones forestales. En un segmento de cada cauce se caracterizó la vegetación de ribera, se midió y georreferenció el material leñoso y se definió la morfología del canal. La vegetación de ribera en Vuelta de Zorra correspondió a renoval-adulto de aproximadamente 200 años con área basal de $69 \mathrm{~m}^{2}$ ha-1 , y en Pichún a un renoval degradado con área basal de $24 \mathrm{~m}^{2} \mathrm{ha}^{-1}$. Considerando el área de cauce lleno como referencia, el volumen de material leñoso en Vuelta de Zorra y Pichún fue 109 y $56 \mathrm{~m}^{3} \mathrm{ha}^{-1}$, respectivamente; los volúmenes de sedimento de fondo atrapado fueron 82 y $30 \mathrm{~m}^{3} \mathrm{~km}^{-1}$, respectivamente, y el 70 y $30 \%$ de este sedimento, respectivamente, se asocia a la presencia de material leñoso. El volumen de material leñoso está asociado al tipo de vegetación de ribera del cauce, el cual es consecuencia del uso histórico del suelo en cada cuenca.

Palabras clave: material leñoso, distribución longitudinal, sedimento de fondo, cauce de montaña.

\section{INTRODUCCIÓN}

Se denomina material leñoso de gran tamaño a todo trozo de madera de diámetro superior a $10 \mathrm{~cm}$ y largo mayor a un metro que se encuentra tanto en los cauces y las llanuras inundables adyacentes (Lenzi et al. 2006). Numerosos estudios demuestran la importancia que tiene la presencia de material leñoso tanto en la morfología como en la ecología del sistema fluvial (Díez et al. 2001, Gurnell et al. 2002).
Los detritos leñosos controlan la morfología del canal en cuencas boscosas, dada su capacidad para formar estructuras como escalones de troncos, grandes acumulaciones de valle y tacos de desviación del flujo (Abbe y Montgomery 2003). La madera bien sea como elemento individual o formando acumulaciones, crean condiciones que favorecen la sedimentación de partículas transportadas por el agua, y en algunas cuencas de montaña, estos sitios pueden almacenar de 10 a 15 veces el volumen del 
transporte anual (May y Gresswell 2003). Andreoli et al. (2007) registran en una cuenca de la Cordillera de Los Andes que el volumen total de sedimento acumulado en el canal, principalmente debido al material leñoso, corresponde aproximadamente al 150 \% de la producción anual de sedimento de la cuenca.

El material leñoso es crucial también para la ecología del corredor fluvial, ya que los troncos y ramas tienen una importante función en el ciclo de nutrientes del ecosistema acuático, al aportar sustrato y alimento para numerosas especies de fauna microbiana e invertebrados, que a su vez son alimento de otras especies como los peces (Bilby y Bisson 1998).

La cantidad y tipo de material leñoso existente en el cauce dependen de las características del bosque que lo alimenta (Comiti et al. 2006, Lenzi et al. 2006). Por ejemplo, los ríos de la costa oeste de EE.UU. y Canadá tienen mayor cantidad de madera que los del centro y este de Norteamérica, lo que se explica por el predominio de bosques de coníferas con árboles de gran tamaño y resistentes a la descomposición. En esta región, algunos ríos de menos de $10 \mathrm{~m}$ de ancho, pueden tener hasta $74 \mathrm{~kg}$ de troncos y acumulaciones de madera por metro cuadrado de cauce, mientras que en otras regiones alcanza un promedio de $4 \mathrm{~kg} \mathrm{~m}^{-2}$ (Bilby y Bisson 1998). Lenzi et al. (2006) indican que el origen del abastecimiento y la cantidad de madera que se almacena en un canal puede ser utilizado como indicador para caracterizar las variaciones tanto de naturaleza morfológica o hidráulica, impuestas por los detritos leñosos sobre este. A su vez, los canales que presentan grandes acumulaciones de madera son de mayor complejidad respecto de aquellos con menor presencia de detritos leñosos.

En América Latina existe poca información específica sobre la abundancia y efectos morfológicos y ecológicos de la presencia de material leñoso en ecosistemas fluviales. En Chile, Vidal Gormaz (1875), luego de sus expediciones de fines del siglo XIX por el sur del país, se refiere a la ensenada Comau, palabra que en lengua huilliche significa "agua que arrastra palos"; Andreoli et al. (2007) informan respecto a la abundancia de detritos leñosos y morfología asociada en dos cauces de la Cordillera de Los Andes. Recientemente, Iroumé et al. (2010) caracterizan el material leñoso en una cuenca ubicada en la Cordillera de la Costa.

Este estudio documenta, por primera vez en Chile, la existencia y características del material leñoso de gran tamaño en dos cuencas con una contrastante historia de uso de suelo, a través de la comparación -de la cantidad y distribución de madera en el cauce- en estas cuencas con diferente cobertura (bosque nativo y plantaciones forestales con especies de rápido crecimiento). Se plantea que la cantidad y características del material leñoso presente en el cauce estarían definidas por el tipo de vegetación de ribera en cada cuenca, y éste a su vez sería consecuencia del uso de la cuenca. Los objetivos de este trabajo son comparar la abundancia, distribución longitudinal y características del material leñoso y volumen de sedimento atrapado; y caracterizar las acumulaciones de madera en función de las piezas que las componen, en los cauces principales de dos cuencas ubicadas en la Cordillera de la Costa.

\section{MÉTODOS}

Área de estudio. El estudio se realizó en dos cuencas, ambas de tercer orden, ubicadas en la Cordillera de la Costa (figura 1), Centro-Sur de Chile. La primera corresponde a la cuenca Vuelta de Zorra, ubicada en la Reserva Coste-

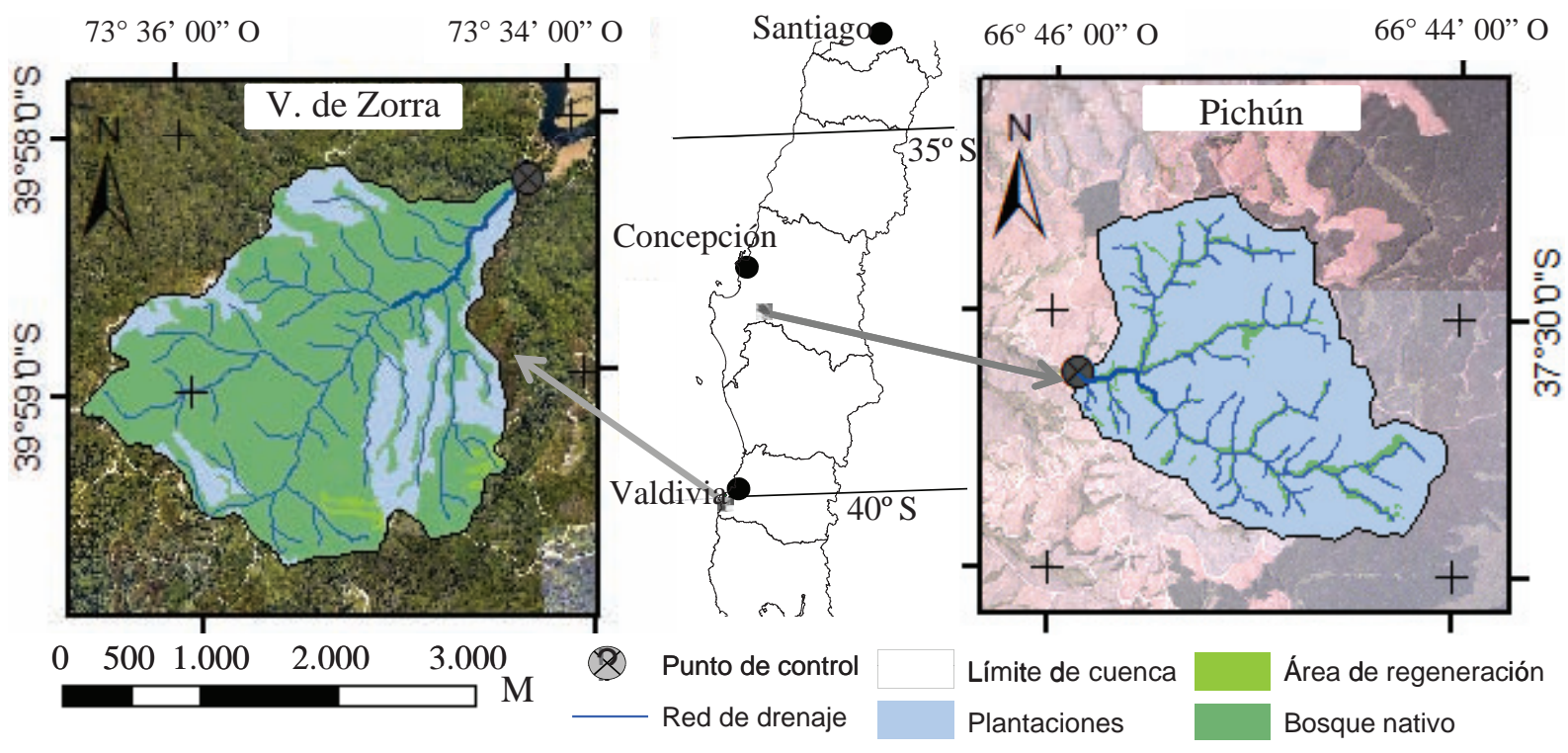

Figura 1. Localización de las cuencas en estudio. Location of study catchments. 
ra Valdiviana en cercanías de la localidad de Chaihuín, a $40 \mathrm{~km}$ al sur-oeste de la ciudad de Valdivia en la región de Los Ríos (3958`12 S; 73 34`13 O). La segunda se localiza en el predio Pichún de propiedad de Forestal Mininco S.A. (en adelante, cuenca Pichún), a $20 \mathrm{~km}$ hacia el sur-oeste de la localidad de Nacimiento en la región del Biobío (37 $30^{`} 12^{\prime} \mathrm{S}$; 66 $66^{\circ} 45^{`} 52^{`} \mathrm{O}$ ).

La cuenca Vuelta de Zorra tiene una superficie de 586,8 ha, pendiente media de la cuenca de $28 \%$, pendiente media del segmento de cauce estudiado de 4,2 \%, tiene forma ovalada y oblonga representada por un índice de compacidad de 1,37. Pichún posee una superficie de 431 ha, pendiente media de 38,2 \% para la cuenca, pendiente media del segmento de cauce estudiado de 9,1 \% y un índice de compacidad de 1,22 que equivale a una forma redonda y ovalada.

La superficie de la cuenca Vuelta de Zorra está cubierta en un $75 \%$ por bosque secundario de entre 150 y 200 años (Iroumé et al. 2010), correspondiente al tipo forestal siempreverde descrito por Donoso (1981), el 24 \% por una plantación de Eucalyptus nitens (Dean et Maiden) de aproximadamente 12 años y el $1 \%$ restante, por varios sectores con una densa regeneración natural. Hacia el este del cauce principal el suelo es arcilloso, derivado de rocas metamórficas mientras que al oeste aparece un suelo arenoso derivado de un basamento granodiorítico (Iroumé et al. 2010). La precipitación media anual en la zona es cercana a los $2.300 \mathrm{~mm}$ (Iroumé et al. 2010).

En la cuenca Pichún el $84 \%$ de la superficie está cubierta por plantaciones de Eucalyptus globulus (Labill.) y el $16 \%$ restante eran caminos y terrenos cubiertos por la vegetación de orilla de la red de drenaje. El suelo en la cuenca es de origen granítico y la precipitación media anual en la ciudad de Nacimiento es de unos $1.400 \mathrm{~mm}$ (Huber et al. 2010).

La precipitación y caudal se midieron en ambas cuencas con sistemas de registro continuo instalados en julio de 2008 en Vuelta de Zorra y en mayo del 2008 en Pichún. En Vuelta de Zorra la estación fluviométrica corresponde una sección natural, mientras que en Pichún está constituida por un marco de hormigón de tipo rectangular de 1,5 m de ancho.

Las cuencas de estudio tienen diferente historial de uso del suelo. La cuenca Vuelta de Zorra se ubica en un área hacia el sur de la desembocadura del río Valdivia con presencia de bosques nativos pertenecientes a los tipos forestales alerce, coigüe de Magallanes y siempreverde (Farías y Teklin 2003). Godoy (s.f) plantea que esta zona presenta evidencias de poblamiento prehispánico, pero que comenzó a ser intervenida de manera más sistemática desde mediados del siglo XIX con la colonización alemana. Desde esa época se desarrolló la industria maderera basada en la explotación, manufactura y venta de madera de alerce (Fitzroya cupressoides (Mol.) Johnston) y otras especies nativas, y luego entre fines de 1980 e inicios de 2000, se desarrolló un proyecto de sustitución de bosque nativo por plantaciones de E. globulus, E. nitens y Eucalyptus delegatensis (R. T. Baker). Desde el año 2003, la cuenca forma parte de la Reserva Costera Valdiviana establecida a través de un consorcio formado por The Nature Conservancy, World Wildlife Fund, Conservation International y otras organizaciones nacionales e internacionales (Farías y Tecklin 2003).

La cuenca Pichún se encuentra en un área donde, según Millán y Carrasco (1993), originalmente predominaban bosques con especies como roble (Nothofagus obliqua (Mirb.) Oerst.), olivillo (Aextoxicon punctatum Ruiz et Pavón), laurel (Laurelia sempervirens (Ruiz et Pavón) Tul.) y lingue (Persea lingue Nees.). Estos bosques fueron degradándose a raíz de talas y quemas extensivas desde el periodo de la conquista y posterior colonización, y luego fueron extensamente eliminados para dar paso a terrenos de cultivos que cubrieron parte importante de la demanda de trigo desde California y Australia, ocurrida a fines del siglo XIX e inicios del XX (Cisternas et al. 1999). A principios de 1950, la zona comenzó a ser reforestada especialmente con Pinus radiata (D. Don) y hacia fines de esa década, la superficie forestada alcanzaba cifras importantes lo que explica la aparición de proyectos industriales como Industrias Forestales S.A. con su planta papelera en la localidad de Nacimiento, que empieza a operar entre 1960 y 1962 (Millán y Carrasco 1993). La cuenca Pichún fue forestada a fines de 1950 y luego de dos rotaciones sucesivas con $P$. radiata, está cubierta con plantaciones de E. globulus establecidas entre los años 2005 y 2008.

Caracterización de la vegetación ribereña en los segmentos estudiados. En Vuelta de Zorra, la caracterización de la vegetación de orilla de cauce se realizó en base a mediciones en cinco parcelas de $500 \mathrm{~m}^{2}$ (50 m de largo en sentido del cauce y $10 \mathrm{~m}$ hacia la ladera) ubicadas a orillas del canal principal. En la parte baja de la cuenca (tramos 1-10), se instaló una parcela al lado izquierdo del cauce, dado que el lado derecho de la ladera está cubierto por pradera y plantación de E. nitens que cubre hasta dos metros del cauce (tramos 1-3 con pradera y 4-10 con orilla derecha plantada), mientras que en la parte media-alta de la cuenca (tramos 11-16) se distribuyeron las otras cuatro parcelas en pares, es decir una a cada lado del cauce para un mismo sitio.

La vegetación de orilla de cauce en Pichún, se caracterizó mediante cinco parcelas de $450 \mathrm{~m}^{2}$, de $30 \mathrm{~m}$ en sentido del canal principal y $15 \mathrm{~m}$ de profundidad. Éstas se ubicaron de forma alternada a la derecha o izquierda del cauce, y fueron distribuidas a lo largo del segmento estudiado en forma relativamente homogénea. Se determinó el ancho de la zona de protección de cauce (la zona no comprometida por las plantaciones comerciales) a cada lado del canal principal en los lugares de localización de las parcelas. El procedimiento, incluyó la medición de esta zona en 10 secciones, considerando desde la orilla del canal hasta que ocurría un cambio visible de la vegetación de la ladera al pasar al área con plantación. 
En ambas cuencas se determinaron las especies vegetales ribereñas y se midió el diámetro ( $>5 \mathrm{~cm}$ ) y altura de los árboles, considerando también aquellos muertos en pie, a los que se les registraron las mismas variables.

Caracterización de los segmentos de los cauces. Entre noviembre de 2008 y marzo de 2009 se estudió un segmento de $1.557 \mathrm{~m}$ de longitud del canal de la microcuenca Vuelta de Zorra y de $1.004 \mathrm{~m}$ en la microcuenca Pichún, en los que se midieron y posicionaron con respecto a puntos de control las piezas de material leñoso y se caracterizaron morfológicamente ambos cauces según la clasificación de Montgomery y Buffington (1997). Los segmentos estudiados fueron divididos en 16 tramos de $97 \mathrm{~m}$ de largo promedio (entre 38 y $143 \mathrm{~m}$ ) para Vuelta de Zorra y en 12 tramos de longitud media de 83,7 m (rango de 27,6 a 134,8 m) para Pichún, definidos según uniformidad de pendiente, sinuosidad, ancho del canal o abundancia del materia leñoso. Estos tramos se numeraron desde aguas abajo (desde el tramo 1 para Vuelta de Zorra y Pichún) hacia aguas arriba (hasta tramo 16 para Vuelta de Zorra y tramo 12 para Pichún). En las orillas de cada cauce se instalaron marcas de referencia (estacas de madera) numeradas para indicar los límites de cada tramo y el posicionamiento de los trozos de madera.

Siguiendo a Iroumé et al. (2010), se levantó el perfil longitudinal de cada tramo usando un distanciómetro laser con inclinómetro para calcular la pendiente de ambos canales. A intervalos no superiores de $15 \mathrm{~m}$, se definieron secciones transversales en cada tramo de cauce para medir el ancho del canal correspondiente al nivel de cauce lleno, entendiéndose este como la altura de la planicie de inundación, y determinar su ancho medio. En cada sección transversal se midió la profundidad en 5-6 perfiles verticales para obtener la profundidad máxima y media correspondiente al nivel de cauce lleno para cada tramo y cauce. Siempre que fue posible, en cada sección se midió además el ancho de la zona de margen.

Toda pieza de madera de diámetro mayor a $10 \mathrm{~cm}$ y longitud superior a un metro que se encontraba en los cauces y orillas adyacentes, fue medida con una cinta métrica y una forcípula para obtener su largo y diámetro medio. La posición se referenció midiendo la distancia y orientación respecto a elementos naturales (rocas, grandes árboles) o las estacas de madera de referencia. En la mayoría de las piezas de madera se colocó una placa metálica numerada (los elementos de grandes dimensiones fueron marcados con hasta cinco placas) para realizar el seguimiento de la movilidad y distancia recorrida a lo largo del cauce.

De cada pieza de material leñoso se registraron las siguientes características: estado de degradación, considerando cuatro niveles (fresco, cuando se notaba presencia de corteza y hojas, secas o no; medio, no había presencia de hojas ni corteza, pero la madera no se notaba porosa ni se deshacía, viejo con presencia de ataques de hongos o de insectos y cuando la madera estaba muy porosa y se desha- cía fácilmente, y vivo para troncos que estaban enraizados a la orilla del cauce), tipo (árbol entero, rama, tronco, tronco con raíz, raíz), especie (agrupados en conífera o latifoliada), posición en el canal (al nivel de cauce lleno, en la margen, dentro del canal pero bajo el nivel de cauce lleno, puente o suspendido sobre el canal y escalón de tronco), orientación con respecto al flujo (paralelo, ortogonal, oblicuo), organización (pieza aislada o en acumulación cuando dos o más piezas estaban en contacto) y origen (residuo de cosecha o madera cortada, derrumbe de laderas, orilla, transportado por la corriente y mortalidad natural). En el caso de las acumulaciones, se midieron las dimensiones geométricas (longitud, ancho y altura desde la base de la acumulación) de cada una y las dimensiones específicas de cada pieza.

El volumen (V) de cada elemento leñoso fue calculado a partir de su diámetro medio (d) y su largo (L), asumiendo una forma sólida cilíndrica mediante la ecuación $\mathrm{V}=\pi \mathrm{d}^{2} \mathrm{~L} / 4$. El volumen de las raíces se aproximó al volumen de un cilindro de diámetro igual a la sección del tallo y de altura igual a la longitud de las raíces, sin considerar su biomasa. El volumen de las acumulaciones se estimó sumando los volúmenes de las piezas que las integraban o atendiendo a sus dimensiones, asumiendo su forma a la de un paralelepípedo cuando no era posible contar todas las piezas que las componían.

A lo largo de los segmentos de los cauces estudiados, se identificó el sedimento de fondo atrapado, considerando el factor de origen, si eran ocasionadas por piezas de madera individual o por acumulaciones, roca o escalones de tronco. El volumen de sedimento se calculó considerando una cuña sólida de dimensiones geométricas medidas con una cinta, sin considerar la porosidad.

La distribución de los volúmenes de madera por tramo se relacionó con las características morfológicas del lecho y superficie incremental de la cuenca, en cada tramo. Se correlacionaron el tamaño de las acumulaciones de madera con las piezas de mayores dimensiones que la componían o piezas clave, estas regresiones fueron probadas a través de Statgraphics ${ }^{\circledR}$ (versión 9.1) con un nivel confianza de $95 \%$.

\section{RESULTADOS}

Caracterización de la vegetación ribereña en los segmentos estudiados. La vegetación de ribera en la sección evaluada de Vuelta de Zorra, correspondió básicamente al mismo tipo de bosque siempreverde descrito para toda la cuenca (Gallo 2010, Iroumé et al. 2010), pero con una mayor dominancia de Myrtaceae como tepú (Tepualia stipularis (H. et A.) Griseb) y, en menor grado, tineo (Weinmannia trichosperma Cav) y trevo (Dasyphyllum diacanthoides (Less.) Cabr). La densidad del bosque de ribera fue de 2.040 árboles $\mathrm{ha}^{-1} \mathrm{y}$ las principales especies fueron luma (Amomyrtus luma (Mol.) Legrand et Kausel), tepa (Laureliopsis philippiana (Looser) Schodde) y canelo (Drimys winteri J. R. et Forster) con 648, 284 y 272 árboles ha-1, 
respectivamente (figura 2). El área basal en esta cuenca fue de 63,1 m ha $\mathrm{ha}^{-1}$ y $69,1 \mathrm{~m}^{2} \mathrm{ha}^{-1}$ al considerar los árboles muertos en pie.

En Pichún la vegetación de ribera corresponde a bosques altamente degradados, derivados del tipo forestal roble-raulí-hualo, que en la actualidad están restringidos a zonas de protección a orilla de cauces. En el segmento estudiado, la vegetación de ribera tuvo en promedio $25 \mathrm{~m}$ de ancho a cada lado del cauce y 662 árboles ha- ${ }^{-1}$, donde roble, peumo (Cryptocarya alba (Mol.) Looser) y olivillo eran las especies con mayor número de pies por hectárea (227, 173 y 89, respectivamente). El área basal fue de 23,1 $\mathrm{m}^{2}$ ha $^{-1} \mathrm{y}$ de $24 \mathrm{~m}^{2}$ ha $^{-1}$ considerando árboles muertos en pie, con el $21 \%$ del área basal $\left(4,9 \mathrm{~m}^{2} \mathrm{ha}^{-1}\right)$ correspondiendo a árboles de $P$. radiata de rotaciones anteriores ubicados en el área de protección de cauce (figura 2).

Características del cauce en los segmentos estudiados en cada cuenca. En general, Vuelta de Zorra presenta mayores dimensiones morfológicas respecto a Pichún (cuadro 1). La pendiente, el ancho y profundidad máxima promedio para el nivel de cauce lleno variaron entre tramos, en ambas cuencas. Vuelta de Zorra presentó como principales estructuras morfológicas: rápidos (riffle; en nueve tramos), rápidos-poza (riffle-pool; en nueve tramos) y escalón-poza

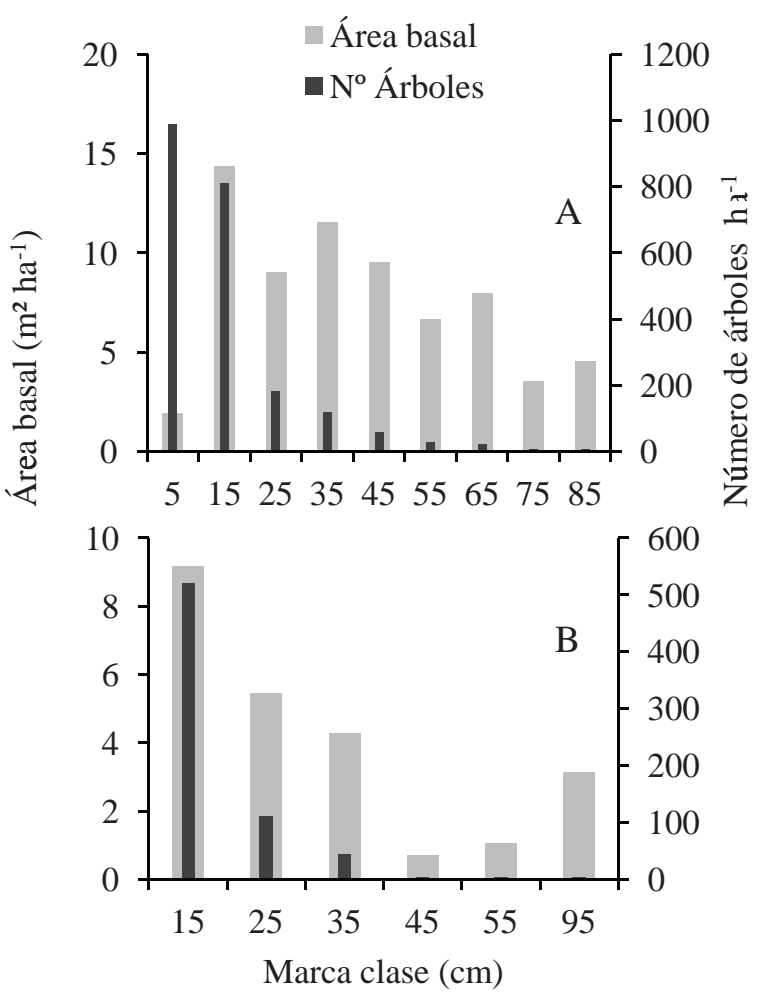

Figura 2. Distribución del área basal $\left(\mathrm{m}^{2} \mathrm{ha}^{-1}\right)$ y número de árboles por hectárea. Vuelta de Zorra (A) y Pichún (B).

Basal area distribution $\left(\mathrm{m}^{2} \mathrm{ha}^{-1}\right)$ and number of trees per hectare. Vuelta de Zorra (A) and Pichún (B) catchment. (step-pool; en cuatro tramos). En Pichún las principales estructuras presentes fueron rápidos (en siete tramos), escalón-poza (en siete tramos) y rápidos-poza (en tres tramos).

Número, volumen, densidad espacial y características del material leñoso. En Vuelta de Zorra se encontraron 484 piezas (311 piezas $\mathrm{km}^{-1}$ ) de los cuales un $80 \%$ (388 piezas) fueron individualizados mediante una o más chapas metálicas. Considerando el canal de cauce lleno como referencia, el volumen medio almacenado en el segmento fue de $109,4 \mathrm{~m}^{3} \mathrm{ha}^{-1} \mathrm{y}$ se evidenció una gran variabilidad desde tres a $378 \mathrm{~m}^{3} \mathrm{ha}^{-1}$ entre tramos (figura 3). En Pichún se cuantificaron 111 piezas $\left(111\right.$ piezas $\left.\mathrm{km}^{-1}\right)$ y de éstas 77 (70 \%) se identificaron con chapas metálicas. El volumen total se estimó en $55,5 \mathrm{~m}^{3} \mathrm{ha}^{-1}$, con una variabilidad entre 0 a $133 \mathrm{~m}^{3} \mathrm{ha}^{-1}$ entre tramos (figura 3). No se encontró relación significativa entre la distribución del número de piezas o volumen y alguna característica del lecho, como ancho plana inundable, pendiente y área incremental en los tramos definidos.

Las dimensiones máximas de los elementos fueron de $80 \mathrm{~cm}$ de diámetro, $24 \mathrm{~m}$ de longitud y 7,7 $\mathrm{m}^{3}$ en volumen para la cuenca Vuelta de Zorra, con un tamaño promedio de las piezas de $24 \mathrm{~cm}$ de diámetro, 5,1 metro de longitud y $0,4 \mathrm{~m}^{3}$ en volumen. Para la cuenca Pichún, se encontró un diámetro de $70 \mathrm{~cm}, 25 \mathrm{~m}$ de largo y un volumen de 2,2 $\mathrm{m}^{3}$ como dimensiones máximas de las piezas, mientras que las dimensiones medias fueron $22 \mathrm{~cm}$ de diámetro, $5,5 \mathrm{~m}$ de largo y un volumen de $0,2 \mathrm{~m}^{3}$. En la figura $4 \mathrm{se}$ muestran los rangos de diámetro y longitud de las piezas individualizadas con chapas metálicas.

Respecto al tipo de elemento, en Vuelta de Zorra el 1,2 \% fueron raíces, 1,2 \% troncos con raíces, 2,5 \% árboles completos, 4,5\% ramas y 90,5\% troncos sin raíces, mientras que en Pichún el 1,8 \% fueron raíces, 5,4 \% árboles completos y 92,8 \% de troncos sin raíces.

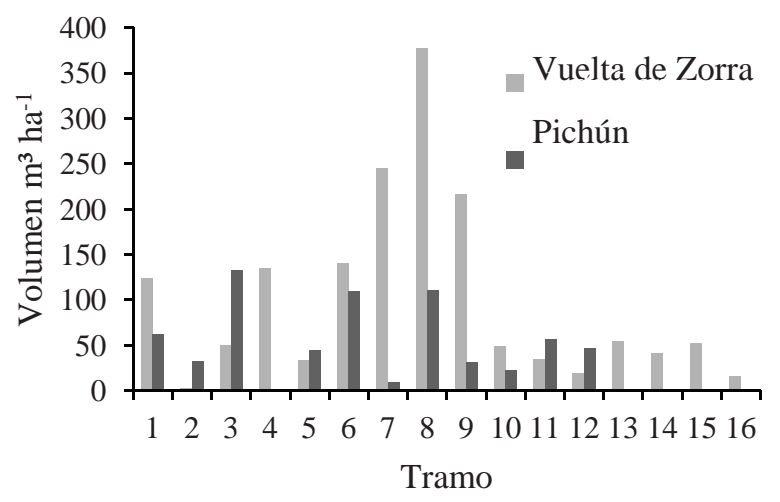

Figura 3. Distribución del volumen en el corredor fluvial para cada tramo.

Volume distribution along the fluvial corridor in each channel segment. 
Cuadro 1. Características de los tramos en cada segmento de cauce estudiado. Reach channel characteristics in each study segment.

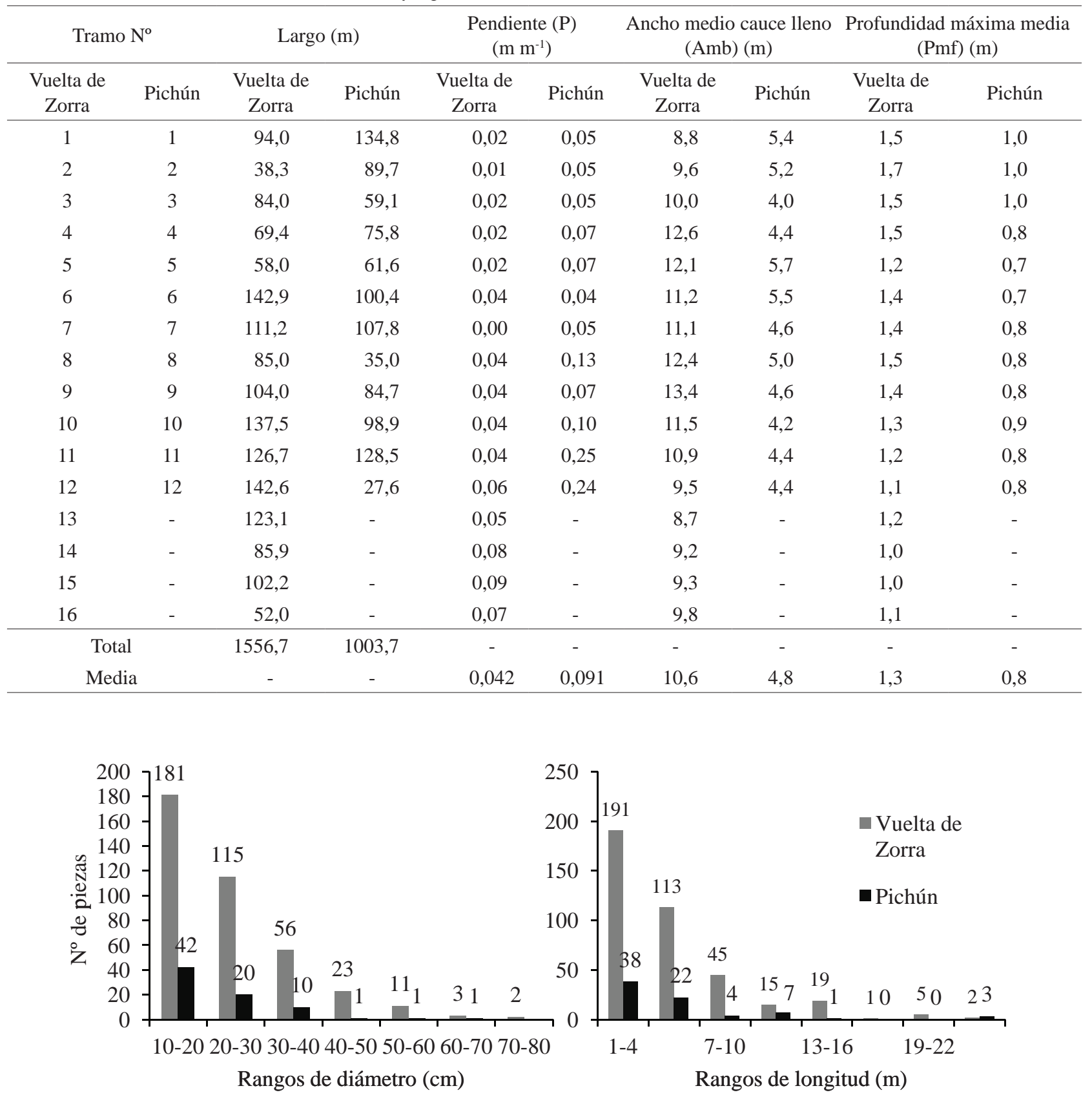

Figura 4. Distribución de la abundancia de material leñoso para las clases de diámetro y longitud del total de elementos marcados con chapas metálicas. Vuelta de Zorra (A), Pichún (B).

Distribution of LW abundance for diameter and length classes of all tagged wood elements, Vuelta de Zorra (A) and Pichún (B).

En relación al estado de degradación, en ambas cuencas la mayoría de las piezas se encontraban en los niveles medio y viejo (figura 5). En Vuelta de Zorra, un alto porcentaje correspondió a especies de latifoliadas mientras que en Pichún se encontró una proporción similar entre latifoliadas y coníferas, posiblemente asociada a la plantación de $P$. radiata cosechada tres años atrás (figura 5).
En Vuelta de Zorra y Pichún la orientación con respecto al flujo fue similar, con un leve predominio de los elementos paralelos al flujo (figura 6). En Vuelta de Zorra, la mayoría parecían haber sido transportados desde aguas arriba por flotación y se ubicaban dentro del cauce del canal de cauce lleno, mientras que en Pichún el origen predominante eran residuos de la cosecha de P. radiata y se ubicaban en las márgenes del canal principal (figura 6). 


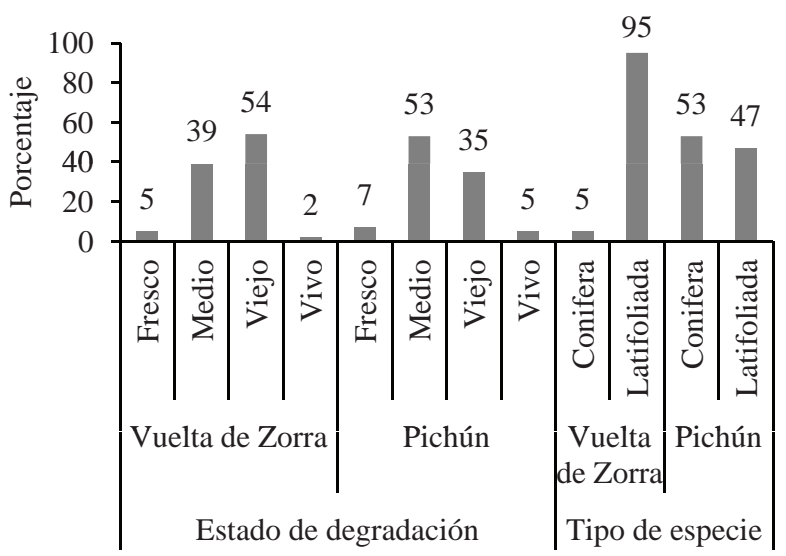

Figura 5. Estado de degradación y tipo de especie de los elementos, para cada cuenca.

Wood piece degradation level and species, each catchment.

Acumulaciones de material leñoso y de sedimentos en cada tramo y cauce. Se encontraron diferencias en el número de troncos acumulados entre las cuencas. En Vuelta de Zorra cerca de un $60 \%$ del volumen total de material leñoso en el segmento estudiado, fueron encontrados formando acumulaciones. En total se encontraron 27 acumulaciones, esto significa aproximadamente una acumulación por cada $58 \mathrm{~m}$ de cauce. Las acumulaciones estaban formadas en promedio por nueve troncos con un volumen promedio de madera contenido en las mismas de 3,96 $\mathrm{m}^{3}$ (entre 0,18 y 20,9 m³). En Pichún las piezas que formaban acumulaciones representaron $8 \%$ del volumen total, que se concentraron en cuatro acumulaciones (una cada $250 \mathrm{~m}$ ). En promedio se registraron tres troncos por acumulación y el volumen medio retenido fue de $0,7 \mathrm{~m}^{3}$ (entre 0,43 y $0,96 \mathrm{~m}^{3}$ ).

Tanto en Vuelta de Zorra como en Pichún, el volumen de las acumulaciones de madera $\left(\mathrm{m}^{3}\right)$ aumentó en forma significativa al aumentar el largo y diámetro máximo de las piezas que la formaban (figura 7).

El volumen de sedimentos acumulados en el canal en Vuelta de Zorra fue mayor que en Pichún. En Vuelta de Zorra se cuantificó un volumen total de $82,1 \mathrm{~m}^{3} \mathrm{~km}^{-1}$ de sedimentos atrapados aguas arriba de piezas individuales de material leñoso, acumulaciones de material leñoso y rocas de gran tamaño que actuaban como cuñas (cuadro 2). Piezas de madera individuales y rocas fueron relativamente similares en cuanto al número de acumulaciones y volumen de sedimento atrapado. Sin embargo, el volumen de sedimento atrapado por las acumulaciones de material leñoso fue menos abundante, pero con un mayor volumen de sedimentos retenidos. En total el sedimento retenido por material leñoso (piezas individuales y en acumulaciones) fue de $87,6 \mathrm{~m}^{3}$.

En Pichún el volumen total de sedimento atrapado fue de $29,8 \mathrm{~m}^{3} \mathrm{~km}^{-1}$, este volumen de sedimento se estableció aguas arriba de escalones de rocas, piezas de material le-
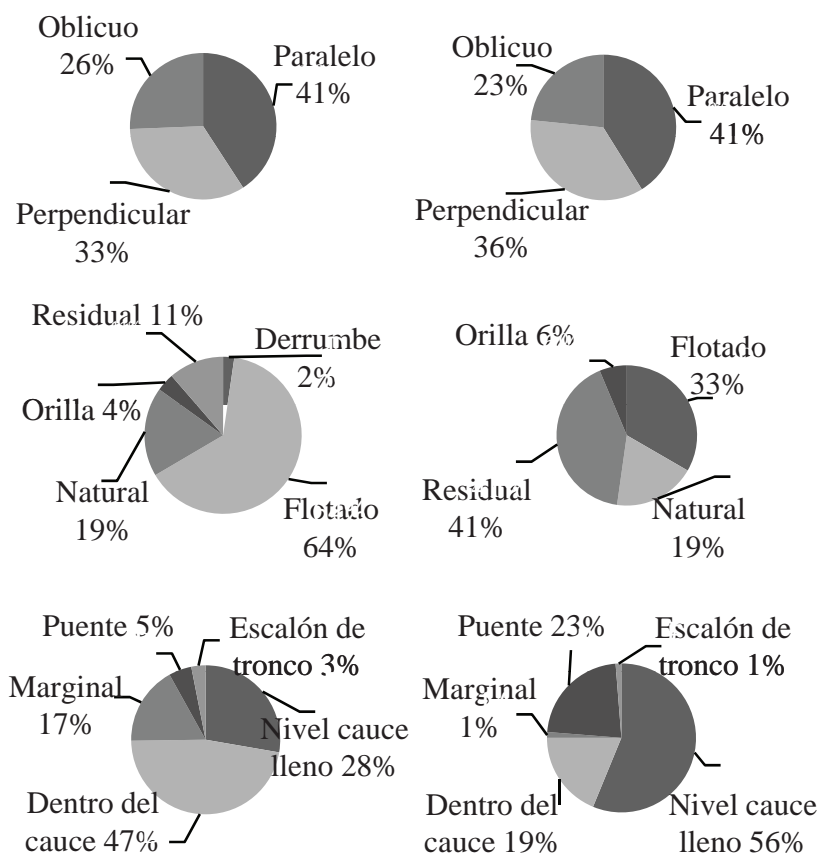

Figura 6. Orientación con respecto al flujo (arriba), origen (al medio) y posición en el canal (abajo) de cada pieza de material leñoso. Vuelta de Zorra (A), Pichún (B).

Orientation to water flow (above), origin (center) and position in the channel (below) of every LW piece. Vuelta de Zorra (A) and Pichún (B).

ñosos, acumulaciones de material leñoso y rocas de gran tamaño. Los escalones de rocas fueron las principales trampas de sedimento con $68 \%$ del volumen total encontrado (cuadro 2). De los otros factores que originaban depósitos de sedimento, las acumulaciones de material leñoso registraron la mayor capacidad de retención con un promedio de 2,5 $\mathrm{m}^{3}$. En total los elementos leñosos (piezas individuales y en acumulaciones) atraparon un volumen de sedimento de $8,9 \mathrm{~m}^{3}$ (cuadro 2).

En Vuelta de Zorra, casi el 70 \% del sedimento atrapado se explica por la presencia de material leñoso, tanto en piezas individuales como en acumulaciones. Sin embargo, en Pichún sólo el 30 \% de los sedimentos está atrapado por estructuras de madera.

\section{DISCUSIÓN}

Características de la vegetación de orilla de cauce en los segmentos estudiados. Las diferencias más relevantes entre las cuencas fue el uso del suelo. La orientación de los cauces, si bien son opuestos, no deberían incidir, principalmente por ser eventos meteorológicos de gran magnitud que influyen en el aporte y pérdida de material leñoso en un segmento de cauce y arrastre de sedimentos en la cuenca. Los tipos forestales presentes en cada cuenca responden principalmente a su ubicación geográfica, mientras que la estructura de la vegetación de cada cuenca depende 

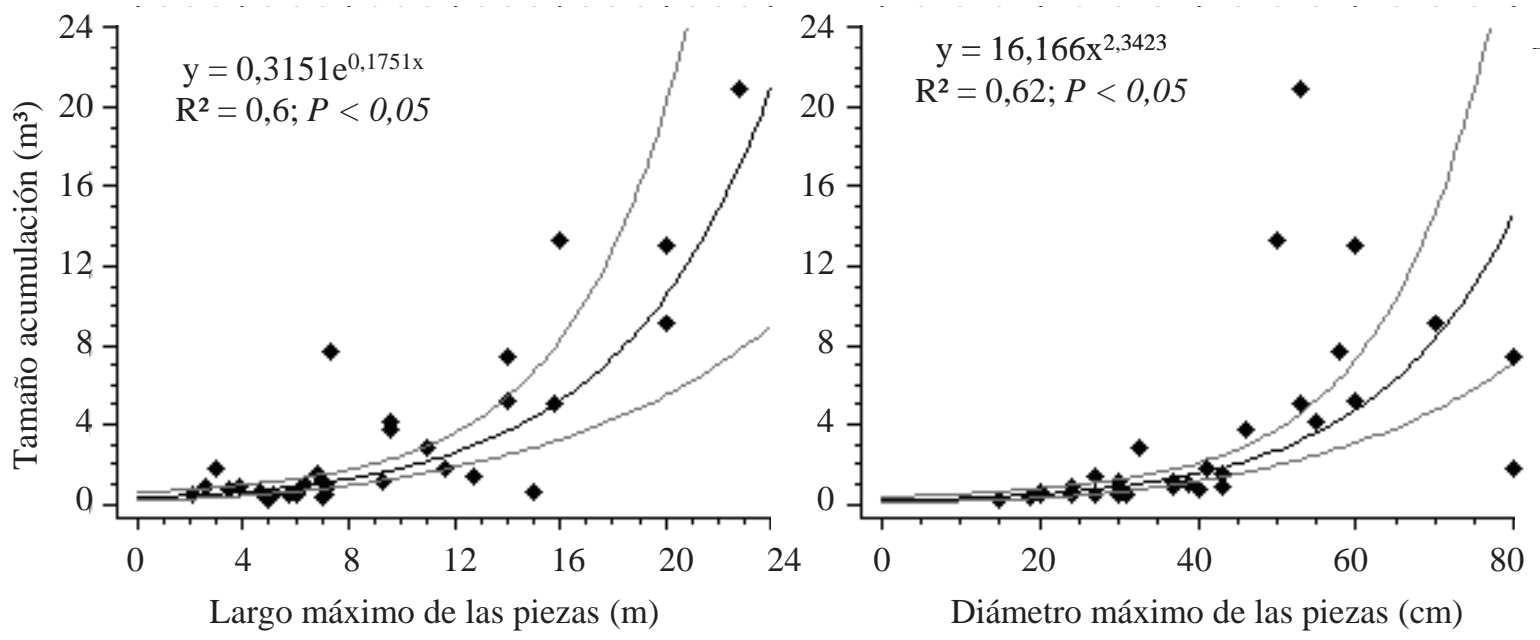

Figura 7. Regresión exponencial e intervalos de confianza (95\%) entre el tamaño de las acumulaciones con la pieza de mayor longitud y mayor diámetro de cada acumulación.

Exponential regression and confidence intervals (95\%), between the size of the log jam and the longest and largest piece of each jam.

Cuadro 2. Fuente de origen y volumen de sedimento atrapado en cada cuenca durante el periodo de estudio. Volume of trapped sediment and sediment trapping sources.

\begin{tabular}{lcccccc}
\hline \multirow{2}{*}{ Fuente de origen } & \multicolumn{3}{c}{ Vuelta de Zorra } & \multicolumn{3}{c}{ Pichún } \\
\cline { 2 - 7 } & $\begin{array}{c}\mathrm{N}^{\text {o }} \text { de } \\
\text { acumulaciones }\end{array}$ & $\begin{array}{c}\text { Volumen } \\
\text { promedio }\left(\mathrm{m}^{3}\right)\end{array}$ & $\begin{array}{c}\text { Volumen total } \\
\left(\mathrm{m}^{3} \mathrm{~km}^{-1}\right)\end{array}$ & $\begin{array}{c}\mathrm{N}^{0} \text { de } \\
\text { acumulaciones }\end{array}$ & $\begin{array}{c}\text { Volumen } \\
\text { promedio }\left(\mathrm{m}^{3}\right)\end{array}$ & $\begin{array}{c}\text { Volumen total } \\
\left(\mathrm{m}^{3} \mathrm{~km}^{-1}\right)\end{array}$ \\
\hline Escalón de rocas & - & - & - & 8 & 2,5 & 20,2 \\
Material leñoso & 10 & 4,1 & 26,4 & 3 & 1,3 & 4,0 \\
Acumulación de material leñoso & 6 & 7,8 & 29,9 & 2 & 2,5 & 4,9 \\
Roca & 9 & 4,5 & 25,8 & 1 & 0,7 & 0,7 \\
Total & 25 & 16,4 & 82,1 & 14 & 7,0 & 29,8 \\
\hline
\end{tabular}

principalmente por el grado de alteración e intensidad de uso del suelo en cada sector. La vegetación de ribera de la cuenca Vuelta de Zorra muestra pocas alteraciones y se clasifica como renovales adultos de aproximadamente 200 años, correspondientes al mismo bosque siempreverde que Iroumé et al. (2010) describen para la cuenca, pero con una mayor dominancia de mirtáceas. En Pichún la vegetación de ribera se deriva de bosques del tipo forestal robleraulí-hualo altamente degradados. El uso histórico de los terrenos en los cuales se ubica esta cuenca, se asocia con la degradación de los bosques originales por talas y quemas extensivas desde el periodo de la conquista y colonización, hasta su posterior eliminación para generar terrenos de cultivos que luego se erosionaron y, finalmente, a la reforestación especialmente con $P$. radiata que se inicia a principios de 1950 (Millán y Carrasco 1993, Cisternas et al. 1999).

Características del material leñoso en los segmentos estudiados. El volumen encontrado en Vuelta de Zorra (109 $\mathrm{m}^{3} \mathrm{ha}^{-1}$ ) se puede comparar con los encontrados en el río El Toro ubicado en la zona andina de la región de La Arauca- nía (117 $\mathrm{m}^{3} \mathrm{ha}^{-1}$; Andreoli et al. 2008) y con el rango entre 100 y $200 \mathrm{~m}^{3} \mathrm{ha}^{-1}$ en bosques maduros no manejados de latifoliadas reportado por Gurnell (2003). Así mismo, este volumen es inferior a los $700 \mathrm{~m}^{3} \mathrm{ha}^{-1}$ encontrados en Tres Arroyos en la cordillera andina de la región de La Araucanía (Andreoli et al. 2007), pero supera a lo informado en canales de segundo-tercer orden en cuencas con bosques manejados en las montañas italianas $\left(30-70 \mathrm{~m}^{3} \mathrm{ha}^{-1}\right.$; Comiti et al. 2006), en cursos de agua de segundo-cuarto orden en los Highlands del Reino Unido (44-88 $\mathrm{m}^{3} \mathrm{ha}^{-1}$; Gurnell et al. 2002), en canales relativamente inalterados de segundo-cuarto orden en Europa Central (20-85 $\mathrm{m}^{3} \mathrm{ha}^{-1}$; Kaczka 2003) y en un cauce de tercer orden de la Península Ibérica (0,4-4,8 $\mathrm{m}^{3} \mathrm{ha}^{-1}$; Díez et al. 2001).

Los $56 \mathrm{~m}^{3} \mathrm{ha}^{-1}$ de material leñoso registrado en Pichún corresponden al menor volumen de material leñoso encontrado en sistemas lóticos chilenos. La medición de la abundancia de este material en Pichún ocurrió entre tres y cuatro años luego de la cosecha del bosque de $P$. radiata que cubría la cuenca desde aproximadamente el año 1987, plantación precedida a su vez por una anterior rotación forestal también de esta especie, por lo que el volumen 
en esta cuenca puede compararse con los registrados en cauces bajo plantaciones de esta conífera. Baillie et al. (1999) reportan en 24 cauces de primer a tercer orden bajo plantaciones maduras (22-34 años) de P. radiata en Nueva Zelandia, una abundancia en un rango entre dos y $345 \mathrm{~m}^{3}$ $\mathrm{ha}^{-1} \mathrm{y}$ una media de $112 \mathrm{~m}^{3} \mathrm{ha}^{-1}$, pero con siete de las 24 cuencas con valores menores que los $56 \mathrm{~m}^{3}$ ha $^{-1}$ medidos en Pichún. En el mismo país y bajo el mismo tipo de plantaciones, Baillie y Davies (2002) entregan la información de otras cinco cuencas donde midieron entre 77 y $200 \mathrm{~m}^{3}$ $\mathrm{ha}^{-1}$ (media de $127 \mathrm{~m}^{3} \mathrm{ha}^{-1}$ ). Por lo tanto, el volumen encontrado en Pichún está en un rango inferior de lo que se ha medido en cauces bajo el mismo tipo de bosques en Nueva Zelandia.

El menor volumen de material leñoso en Pichún que en Vuelta de Zorra se puede explicar por las diferencias en el área basal de los bosques de ribera $\left(24 \mathrm{~m}^{2} \mathrm{ha}^{-1}\right.$ para Pichún y $69 \mathrm{~m}^{2}$ ha $^{-1}$ para Vuelta de Zorra) y a la menor área potencial de suministro de madera en Pichún, que está limitada por el ancho de $25 \mathrm{~m}$ que tiene la vegetación ribereña a cada lado del cauce; mientras que en Vuelta de Zorra, está asociada a la altura de los árboles que al caer pueden alcanzar el cauce (por ejemplo, árboles de coihue que pueden alcanzar $35 \mathrm{~m}$ de altura extendiendo hasta esta distancia el área de suministro en Vuelta de Zorra). Adicionalmente, la menor durabilidad de la madera de $P$. radiata que caracteriza a la cuenca de Pichún frente a los Nothofagus spp. de Vuelta de Zorra (Garrett et al. 2008), permite asegurar que es difícil que en la primera se encuentren trozos de madera de la rotación anterior, mientras que en la segunda se genera una mayor acumulación, de los últimos 50 -70 años. Finalmente, el manejo forestal puede ser también un factor que influye en el menor volumen de madera encontrado en Pichún, ya que se encontraron menos elementos claves de grandes dimensiones, capaces de atrapar elementos leñosos más pequeños (Meleason et al. 2002).

Tanto en Pichún como en Vuelta de Zorra, el porcentaje de elementos orientados en forma paralela a la corriente fue significativamente más alto que si las piezas hubieran estado orientadas aleatoriamente, mientras que fueron menores las piezas orientadas de manera oblicua. La distribución de las orientaciones fue similar a la encontrada por Baillie et al. (2008) en el río Whirinaki en Nueva Zelandia. Andreoli et al. (2008) reportan que en el río Toro, Chile, las piezas no estaban distribuidas aleatoriamente, pero en este caso los elementos dispuestos ortogonalmente a la corriente representan un $51 \%$.

El uso del suelo parece definir las diferencias en el origen de las piezas de madera que se encuentran en el cauce de ambas cuencas estudiadas. Así, en la cuenca Vuelta de Zorra, cuyos bosques tiene menor intervención antropogénica, la mayoría de los elementos tiene origen "flotado", es decir, que fueron arrastrados desde aguas arribas al punto en que se registraron al momento de la medición. Por otra parte, el origen residual de los elementos leñosos (restos de cosecha o intervenciones) se concentra principalmente en Pichún, donde los de origen flotado corresponden a un segundo orden en términos de abundancia. En otros estudios en cuencas con bosques no manejados también se ha reportado que la causa más probable de aporte de madera, es el transporte desde aguas arriba, aunque con porcentajes ligeramente mayores. Andreoli et al. (2007) encuentran un $88 \%$ de elementos flotados en Tres Arroyos en Los Andes (Chile) y Mao et al. (2008) un 75 \% en el arroyo Buena Esperanza en Tierra del Fuego (Argentina).

Las posiciones "dentro del cauce" y "marginal" (respecto del borde del canal) concentran en conjunto un $64 \mathrm{y}$ 69 \% de las piezas para Vuelta de Zorra y Pichún, respectivamente. Las diferencias entre estas clasificaciones para una u otra cuenca, pueden atribuirse a las características propias del canal, como pendiente y ancho, que afectan el depósito de madera en el cauce. Así la menor pendiente y el mayor ancho del cauce en Vuelta de Zorra explica que la mayoría de los elementos se encuentren dentro del cauce, mientras que en Pichún, al presentar un canal con mayor pendiente y más angosto, existe mayor depósito de material leñoso hacia la zona marginal. A estas características parecen también responder los elementos posicionados en la zona de nivel de cauce lleno. Las posiciones como puente y escalón de troncos dependen de la relación entre el tamaño de los elementos leñosos y las dimensiones de ancho de cauce; por lo tanto, el menor número de elementos como puente en Vuelta de Zorra, se relaciona con un menor valor de la razón longitud media de los elementos/ ancho medio del cauce $(5,1 / 10,6=0,48)$ en contraste a $(5,5 / 4,8=1,2)$ para la cuenca de Pichún. En Tres Arroyos (Andreoli et al. 2007) más de la mitad de los elementos ocurre en las márgenes (52 \%) mientras que dentro del canal se encuentra un $23 \%$, esto asociado a la fuerte inestabilidad de las laderas del canal.

Acumulaciones de material leñoso y de sedimentos en cada tramo y cauce. El número de acumulaciones o tacos formados por el material leñoso en Vuelta de Zorra son mucho menores que los encontrados en Tres Arroyos por Andreoli et al. (2007) con valores de 51 tacos km-1 con un espaciamiento entre ellos de $20 \mathrm{~m}$, pero similares a los espaciamientos de 25-50 m encontrados por Martin y Benda (2001) para áreas de tamaño similar (0-10 km²) en la cuenca del Game Creek, Alaska. Para Vuelta de Zorra y Pichún, las acumulaciones estaban formadas en promedio por nueve y tres troncos, respectivamente, con un volumen medio de madera contenido en los mismos de 3,96 y 0,7 $\mathrm{m}^{3}$. Martin y Benda (2001) también registran acumulaciones de entre cinco y 15 troncos en cuencas de hasta $10 \mathrm{~km}^{2}$, pero estos valores son menores a las registrados por Andreoli et al. (2007) donde estaban formados, en promedio por 13 troncos, mientras que el volumen medio de madera contenido en las mismas era de $9 \mathrm{~m}^{3}$. Respecto a la relación positiva que se obtuvo entre el tamaño de las acumulaciones con el diámetro y largo del elemento de mayor tamaño, relación similar es reportada Mao et al. (2008), 
encontrando una correlación positiva $(r=0,62 ; P<0,001)$ entre la altura de escalones de tronco y el diámetro medio de los elementos que la constituían.

El mayor volumen de sedimentos atrapados en Vuelta de Zorra $\left(82 \mathrm{~m}^{3} \mathrm{~km}^{-1}\right)$ respecto a Pichún $\left(30 \mathrm{~m}^{3} \mathrm{~km}^{-1}\right)$ responde principalmente a lo planteado por Abbe y Montgomery (2003) en que el potencial de un canal para atrapar y almacenar sedimentos está controlado principalmente por el volumen de material leñoso. Esta tendencia se comprueba con los resultados obtenidos por Andreoli et al. (2007), quienes encontraron un volumen de sedimentos retenido de $1.300 \mathrm{~m}^{3} \mathrm{~km}^{-1}$ en Tres Arroyos donde el volumen de madera en el cauce corresponde a $700 \mathrm{~m}^{3} \mathrm{ha}^{-1}$.

\section{CONCLUSIONES}

Se encontró que el volumen de material leñoso en el cauce de la cuenca Vuelta de Zorra duplica lo encontrado en la cuenca Pichún y esta diferencia está asociada principalmente a las dimensiones y características de la vegetación presente en el área de potencial suministro de madera al cauce. Las características de la vegetación a orilla de cauce derivan esencialmente del grado de alteración e intensidad de uso de suelo al que se ha sometido la cuenca a lo largo del tiempo.

En Vuelta de Zorra se encontró que la cantidad de elementos leñosos formando acumulaciones es superior, lo que está asociado principalmente a la mayor cantidad de madera en este canal y que el tamaño de estas acumulaciones tiene una directa relación con el tamaño de los elementos leñosos claves que la componen. Estos elementos y principalmente aquellos que se encuentran formando acumulaciones tienen un rol importante en la retención y almacenamiento de sedimento de fondo.

\section{AGRADECIMIENTOS}

Esta investigación se realizó en el marco del Proyecto FONDECYT 1080249. Los autores agradecen a The Nature Conservancy y Forestal Mininco S.A. por su interés y apoyo en los estudios que se están realizando en las cuencas Vuelta de Zorra y Pichún, respectivamente.

\section{REFERENCIAS}

Abbe T, D Montgomery. 2003. Patterns and processes of wood debris accumulation in the Queets river basin, Washington. Geomorphology 51: 81-107.

Andreoli A, G Carlig, F Comiti, A Iroumé. 2007. Residuos leñosos de gran tamaño en un torrente de la Cordillera de los Andes, Chile: su funcionalidad e importancia. Bosque 28(2): 83-96.

Andreoli A, F Comiti, L Mao, A Iroumé, MA Lenzi. 2008. Evaluación de los volúmenes y de los efectos hidro-morfológicos del material leñoso en dos torrentes andinos (Chile). Ingeniería del Agua 15(3): 189-204.
Baillie BR, L Cummins, MO Kimberley. 1999. Measuring woody debris in the small streams of New Zealand's pine plantations. New Zealand Journal Marine and Freshwater Resources 33: 87-97.

Baillie BR, TR Davies. 2002. Influence of large woody debris on channel morphology in native forest and pine plantation streams in the Nelson region, New Zealand. New Zealand Journal Marine and Freshwater Resources 36: 763-774.

Baillie B, L Garrett, A Evanson. 2008. Spatial distribution and influence of large woody debris in an old-growth forest river system, New Zealand. Forest Ecology and Management 256: 20-27.

Bilby R, P Bisson. 1998. Function and distribution of large woody debris in Pacific coastal streams and rivers. In Naiman RJ, RE Bilby eds. River Ecology and Management Lessons from the Pacific Coastal Ecoregion. New York, USA. Springer. p. 324-346.

Cisternas M, P Martínez, C Oyarzun, P Debels. 1999. Caracterización del proceso de reemplazo de vegetación nativa por plantaciones forestales en una cuenca lacustre de la Cordillera de Nahuelbuta, VIII Región, Chile. Revista Chilena de Historia Natural 72: 661-676.

Comiti F, A Andreoli, MA Lenzi, L Mao. 2006. Spatial density and characteristics of woody debris in five mountain rivers of the Dolomites (Italian Alps). Geomorphology 78 (1-2): 44-63.

Díez J, A Elosegi, J Pozo. 2001. Woody debris in North Iberian stream: influence of geomorphology, vegetation, and management. Environmental Management 28: 687-698.

Donoso C. 1981. Tipos forestales de los bosques nativos de Chile. Investigación y Desarrollo Forestal. Santiago, Chile. CONAF-FAO. 70 p. (Documento de Trabajo $N^{\circ} 38$ ).

Farías A, Tecklin D. 2003. Caracterización preliminar de los predios Chaihuín- Venecia, Cordillera de La Costa Décima Región. Valdivia, Chile. Serie de Publicaciones WWF Chile, Programa Ecoregión Valdiviana. 25 p. (Documento Nº 6).

Garrett LG, GR Oliver, SH Pearse, MR Davis. 2008. Decomposition of Pinus radiata coarse woody debris in New Zealand. Forest Ecology and Management 255: 3839-3845.

Godoy M, s.f. Los asentamientos humanos del borde costero de la provincia de Valdivia Sur: El caso del predio Chaihuín Venecia. Valdivia, Chile. Dirección Museológica Universidad Austral de Chile. 55 p.

Gurnell A, H Piégay, F Swanson, S Gregory. 2002. Large wood and fluvial processes. Freshwater Biology 47: 601-619.

Gurnell A. 2003. Wood storage and mobility. In Gregory SV, KL Boyer, AM Gurnell eds. The ecology and management of wood in world rivers. Bethesda, Maryland, USA. American Fisheries Society. p. 75-91.

Huber A, A Iroumé, C Mohr, C Frêne. 2010. Efecto de las plantaciones de Pinus radiata y Eucalyptus globulus sobre el recurso agua en la Cordillera de la Costa de la región del Biobío, Chile. Bosque 31(3): 219-230.

Iroumé A, A Andreoli, F Comiti, H Ulloa, A Huber. 2010. Large wood abundance, distribution and mobilization in a third order Coastal mountain range river system, southern Chile. Forest Ecology and Management 260: 480-490.

Kaczka RJ. 2003. The coarse woody debris dams in mountain streams of central Europe, structure and distribution. Studia Geomorphologica Carpatho-Balcanica 38: 111-127.

Lenzi MA, F Comiti, L Mao, A Andreoli, E Pecorari, E Rigon. 
2006. El control de detritos leñosos y el manejo de la vegetación en el cauce. Padova, Italia. Universita’ degli studi di Padova. 185 p.

Mao L, S Burns, F Comiti, A Andreoli, A Urciuolo, M GaviñoNovillo, R Iturraspe, MA. Lenzi. 2008. Acumulaciones de detritos leñosos en un cauce de montaña de Tierra del Fuego: análisis de la movilidad y de los efectos hidromorfológicos. Bosque 29 (3): 197-211.

Martin DJ, LE Benda. 2001. Patterns of instream wood recruitment and transport at the watershed scale. Transaction of the American Fisheries Society 130: 940-958.

May C, R Gresswell. 2003. Processes and rates of sediment and wood accumulation in headwater streams of the Oregon Coast range, USA. Earth Surface Processes and Landforms 28: 409-424.

Meleason M, S Gregory, J Bolte. 2002. Simulation of stream wood source distance for small streams in the western cascades, Oregon. USDA Forest Service General Technical Report PSW-GTR 181: 457-466.

Millán J, P Carrasco. 1993. La forestación en la VIII Región. In Faranda F, O Parra eds. Proyecto Eula-Chile. Elementos cognoscitivos sobre el recurso suelo y consideraciones generales sobre el ordenamiento agroforestal. Concepción, Chile. Universidad de Concepción. p. 75-84. (Serie Propuestas de ordenamiento Vol. 4).

Montgomery DR, JM Buffington. 1997. Channel-reach morphology in mountain drainage basins. Geological Society of America Bulletin 109: 596-611.

Vidal Gormaz F. 1875. Reconocimiento del Río Maullín, por la Comisión Esploradora de Chiloé i Llanquihue. Anuario Hidrográfico de la Marina de Chile. Valparaíso, Chile. Instituto Hidrográfico de la Armada de Chile. p. 167-262.

Recibido: 12.11 .10

Aceptado: 26.08.11 
